\title{
La Ligue de l'enseignement (1902-1904) : héritage du passé et nouveaux défis
}

\section{Ruby Heap}

Volume 36, numéro 3, décembre 1982

URI : https://id.erudit.org/iderudit/304067ar

DOI : https://doi.org/10.7202/304067ar

Aller au sommaire du numéro

\section{Éditeur(s)}

Institut d'histoire de l'Amérique française

\section{ISSN}

0035-2357 (imprimé)

1492-1383 (numérique)

Découvrir la revue

\section{Citer cet article}

Heap, R. (1982). La Ligue de l'enseignement (1902-1904) : héritage du passé et nouveaux défis. Revue d'histoire de l'Amérique française, 36(3), 339-373.

https://doi.org/10.7202/304067ar d'utilisation que vous pouvez consulter en ligne.

https://apropos.erudit.org/fr/usagers/politique-dutilisation/ 


\title{
LA LIGUE DE L'ENSEIGNEMENT (1902-1904): HÉRITAGE DU PASSÉ ET NOUVEAUX DÉFIS*
}

\author{
RUBY HEAP \\ Département d'histoire \\ Université de Montréal
}

À l'exception des travaux de pionnier de Louis-Philippe Audet ${ }^{1}$, l'histoire de l'éducation au Québec n'a pas donné lieu à une production abondante. Ce n'est qu'en 1978 que fut publié le premier ouvrage de synthèse consacré au collège classique, jusqu'à très récemment l'une des institutions-clefs du système scolaire québécois $^{2}$. Par ailleurs, ce que les historiens de l'éducation appellent «l'éducation nouvelle» n'a pas encore fait au Québec l'objet de recherches comparables, sur le plan de la démarche et de la problématique, à celles effectuées jusqu'à ce jour au Canada anglais. Ainsi, il reste à analyser de façon systématique l'impact du processus industriel et urbain sur les programmes, les structures et l'administration du système éducatif québécois ${ }^{3}$. Le terrain d'enquête est donc neuf, et des interrogations se posent toujours sur les rapports entre l'institution scolaire et la société québécoise au moment où cette dernière subit d'importantes mutations économiques et sociales.

L'une de ces interrogations concerne la nature du mouvement d'opinion qui, au tournant du siècle, se met à réclamer d'une manière articulée une série de réformes dans le domaine de l'éducation. Nous avons donc choisi de nous pencher sur ce sujet, par le biais d'une étude de la Ligue de l'Enseignement, une association fondée en 1902, dans le but spécifique de soulever la question scolaire au Québec et d'apporter des changements au système éducatif. En examinant l'organisation, la composition et le programme de la Ligue, il sera possible de repérer les agents sociaux qui s'intéressent alors à la réforme scolaire, puis de définir leurs préoccupa-

* Texte d'une communication présentée en février 1982 à l'Université de Toronto lors du Congrès sur "L'Éducation nouvelle au Canada, 1880-1930», organisé sous les auspices de l'Association canadienne d'histoire de l'éducation.

Son Histoire de l'enseignement au Québec (Montréal, 1971, 2 vols.) demeure le meilleur ouvrage de synthèse que nous possédions sur le sujet.

2 Claude Galarneau, Les collèges classiques au Canada français (Montréal, 1978).

3 C'est ce que nous avons entrepris de faire dans le cadre de notre recherche doctorale qui couvre la période 1898-1920. Notre étude examinera aussi l'évolution des rapports de pouvoir entre les appareils politique et religieux au sein du système scolaire public québécois.

RHAF, vol. 36, no 3, décembre 1982 
tions majeures à cet égard. Pour terminer, nous considérerons les revendications formulées par la Ligue et ses promoteurs en rapport avec l'évolution de l'enseignement au Québec sous le régime libéral de Lomer Gouin (1905-1920), période particulièrement fertile en réalisations dans le secteur éducatif. Bien que nous ne puissions analyser en détail les divers processus qui dictèrent cette évolution, notre démarche permettra de préciser lesquelles des réformes préconisées par la Ligue étaient acceptables et applicables dans le contexte politico-religieux de l'époque.

\section{I - La province de Québec au début du XXe siècle: le cadre conjoncturel}

L'origine de la Ligue de l'Enseignement est largement reliée aux changements d'ordre conjoncturel qui se produisent au Québec au tournant du siècle. Sur le plan économique, la province est entrée dans une période de prospérité qui engendre un climat d'optimisme au sein des milieux politiques et d'affaires. L'industrialisation connaît une phase de développement rapide et franchit une étape décisive avec l'apparition de nouvelles industries basées sur l'exploitation des richesses naturelles. Ce processus se reflète dans la répartition de la main-d'oeuvre: dès le recensement de 1901, la population active employée dans les occupations secondaires et tertiaires dépasse celle du secteur primaire ${ }^{4}$. Il faut souligner, par contre, que le développement économique du Québec est financé de plus en plus par des capitaux étrangers (britanniques et américains), ce qui accentue l'état de dépendance et de satellisation de la province.

L'industrialisation rapide du Québec se traduit par un taux d'urbanisation croissant. En 1901, un peu plus d'un tiers des Québécois habitent déjà les villes, et le recensement de 1921 confirmera le caractère majoritairement urbain de la province ${ }^{5}$. C'est Montréal qui domine nettement le paysage urbain du Québec. L'île de Montréal regroupe $52,9 \%$ de la population urbaine de la province en $1901^{6}$. La ville draîne une part de plus en plus considérable des Québécois ruraux qui abandonnent les campagnes dans l'espoir d'un sort meilleur. En 1901, les francophones rassemblent $63,9 \%$ de la population de l'île de Montréal, alors que les Britanniques en regroupent $31,6 \%{ }^{7}$.

4 William Larkin et Patrick Allen, Tendances occupationnelles au Canada (Montréal, École des Hautes Études commerciales, 1951), étude no 4: 52.

5 L.O. Stone, Urban Development in Canada (Ottawa, Dominion Bureau of Statistics, 1967), 29. En 1921, le taux d'urbanisation de la province atteint $51,8 \%$.

6 Paul-André Linteau, René Durocher et Jean-Claude Robert, Histoire du Québec contemporain (Les Éditions du Boréal Express, 1979), 411.

7 Ibid., 63, tableau 6. 
Par ailleurs, Montréal assume pleinement sa fonction de métropole industrielle, commerciale et financière. En 1900, la valeur de sa production industrielle représente $55 \%$ de la production totale du Québec ${ }^{8}$. La ville se situe toujours à la tête des grands réseaux ferroviaires; elle joue en plus le rôle de principal port du Canada et demeure le centre financier du pays. Toute cette activité économique est dirigée par une grande bourgeoisie qui oeuvre à l'échelle canadienne, et même à l'échelle internationale. Cette classe massivement anglophone est concentrée dans la métropole. Quoique les Canadiens français y soient nettement sous-représentés, un bon nombre d'entre eux exercent une action économique importante au niveau régional et local, que ce soit dans le secteur financier, industriel ou foncier. Ces représentants de la moyenne bourgeoisie québécoise, dont on a traditionnellement négligé l'importance ${ }^{9}$, se concentrent également à Montréal et leur pouvoir s'étend dans les domaines politique et social. Comme nous le verrons plus loin, ces bourgeois canadiens-français s'intéressent de près aux questions d'enseignement.

La poussée de l'industrialisation et de l'urbanisation, amorcée au tournant du siècle, coïncide, sur le plan politique, avec l'arrivée du parti libéral au pouvoir. L'élection de F.-G. Marchand en 1897 met fin au long règne des conservateurs qui dominaient la scène politique depuis la Confédération. Or, la question scolaire constitue l'une des préoccupations majeures du nouveau gouvernement. Depuis plusieurs années, la presse libérale réclame avec insistance une réforme de l'enseignement. Elle dénonce l'état précaire du système scolaire primaire et fait également le procès de l'enseignement secondaire classique, qu'elle accuse d'être mal adapté aux besoins du temps. Cette campagne est surtout menée par une poignée de libéraux dits "radicaux» ou "avancés» qui aspirent à une laïcisation graduelle du système éducatif. Ces réformateurs se déclarent partisans du contrôle de l'État sur l'enseignement et de l'instruction gratuite et obligatoire ${ }^{10}$. Ils prolongent une tradition anticléricale qui remonte au milieu du XIXe siècle, époque où les Rouges, inspirés par le mouvement libéral européen, ont fait campagne pour la déconfessionnalisation de l'école et l'établissement d'un système d'enseignement laïc ${ }^{11}$. Comme leurs prédécesseurs,

Ibid., 414.

$9 \quad$ Sur cette question, voir l'article de Paul-André Linteau, «Quelques réflexions autour de la bourgeoisie québécoise, 1850-1914», Revue d'histoire de l'Amérique française, 30, 1 (juin 1976): 55-66.

10 Voir R. Heap, «L'Église, l’État et l'éducation au Québec, 1875-1898», thèse de maîtrise (Montréal, McGill University, 1979), 361 ss.

11 Sur la pensée éducative des Rouges, il faut consulter l'ouvrage de Jean-Paul Bernard, Les Rouges: libéralisme, nationalisme et anticléricalisme au milieu du XIXe siècle (Montréal, 1971). 
les radicaux espèrent affranchir l'institution scolaire de la tutelle ecclésiastique. En plus de monopoliser l'enseignement secondaire et supérieur, l'Église exerce aussi son influence dans le secteur primaire par l'entremise du Comité catholique du Conseil de l'Instruction publique, où siègent comme membres ex officio tous les évêques de la province.

$\mathrm{Au}$ début du XXe siècle, le principal foyer radical est localisé à Montréal. Ses effectifs sont peu nombreux, mais il abrite néanmoins des membres en vue de la petite bourgeoisie francophone: journalistes, avocats, médecins, échevins. Afin de propager leurs idées, ces individus ont même fondé en 1896 une société maçonnique, la loge L'Émancipation, dont la charte origine directement du Grand-Orient de France. La loge place en tête de ses objectifs immédiats le rétablissement du ministère de l'Instruction publique, aboli par les conservateurs en 1875, alors même que l'Ontario mettait le sien sur pied ${ }^{12}$. Sur ce point, les libéraux radicaux rencontrent les vues du gouvernement Marchand, qui compte dans ses rangs des réformateurs, tel le secrétaire provincial, Joseph-Emery Robidoux. En décembre 1897, celui-ci soumet à l'Assemblée législative un projet de loi qui substitue un ministre au surintendant de l'Instruction publique, dont le poste revêtait un caractère inamovible. Les implications du bill sont claires: contrôle du pouvoir civil sur l'enseignement et élimination du principe de «l'éducation au-dessus de la politique», sur lequel est fondé depuis 1875 le système scolaire québécois. Or, l'épiscopat est fermement résolu à prévenir une rupture de l'équilibre Église-État qu'engendrerait le rétablissement d'un ministère. Bien que le bill soit adopté à l'Assemblée législative, il est défait par la suite au Conseil législatif, où les conservateurs ultramontains championnent une politique antiétatique en matière d'éducation. Lorsque S.-N. Parent succédera à Marchand en 1900, il ne ranimera pas la mesure controversée de son prédécesseur.

Cette reculade du gouvernement québécois sur la question du ministère de l'Instruction publique illustre l'état du rapport de force existant entre le parti libéral et l'épiscopat dans le domaine éducatif au tournant du siècle. Mais l'apaisement politico-religieux qui s'ensuit n'étouffe pas l'agressivité des radicaux qui conservent l'espoir de rallier le gouvernement libéral à leur cause. On ne s'étonnera donc pas de les retrouver au sein de la Ligue de l'Enseignement, nouvelle association mise sur pied à Montréal à l'automne 1902.

12 Robert Rumilly, Histoire de la province de Québec (Montréal, Éditions Bernard Valiquette), 9: 19. 


\section{II - Organisation et composition de la Ligue de l'Enseignement}

Le 9 octobre 1902, une centaine de citoyens montréalais se réunissent au théâtre Delville afin de jeter les bases d'une Ligue de l'Enseignement destinée à éclairer les pouvoirs publics et la population sur les avantages qu'apporteraient certains changements dans le système scolaire. L'assemblée, composée majoritairement d'échevins, d'avocats, de médecins et de journalistes, ne compte que très peu de professionnels de l'enseignement, bien qu'on remarque la présence de l'inspecteur d'écoles Bernard Lippens et celle de Napoléon Brisebois, professeur à l'École normale JacquesCartier. Le secrétaire de l'assemblée est Godfroy Langlois, rédacteur en chef de La Patrie, organe du parti libéral dans la métropole. Héritier de la tradition «rouge», Langlois dirige aussi la loge L'Émancipation, toujours active malgré ses effectifs très modestes ${ }^{13}$. Selon lui, la nouvelle association contribuera à réduire l'apathie générale qui règne au Québec au sujet de l'instruction publique et remplira dans le secteur éducatif un rôle équivalent à celui des chambres de commerce dans le domaine économique. La Ligue de l'Enseignement, prend soin de préciser Langlois, n'est hostile à personne et n'est fondée pour combattre qui que ce soit. C'est pourquoi, ajoute-t-il, le gouvernement provincial et le Conseil de l'Instruction publique auront grand intérêt à prendre contact avec l'association, qui n'aspire qu'à promouvoir la cause de l'éducation au Québec.

L'élection des officiers vient clore la séance de fondation de la Ligue. Olivier Faucher, commerçant et ex-échevin, est élu président, et Godfroy Langlois, vice-président. Le notaire Victor Lamarche remplira les fonctions de trésorier. Deux secrétaires sont également choisis: le professeur Brisebois et le journaliste Arthur Beauchesne, rédacteur du quotidien conservateur montréalais $L e$ Journal. Aux nouveaux officiers est alors confié le soin de dresser les règlements et la constitution de la Ligue ${ }^{14}$.

Cette dernière est soumise et adoptée lors d'une seconde assemblée tenue le 20 novembre suivant. Le document stipule que le but de la Ligue est de «provoquer dans la province de Québec, par tous les moyens possibles, l'initiative individuelle au profit du développement de l'instruction publique». Pour atteindre cet objectif, l'association aura principalement recours aux publications, aux conférences et aux discussions publiques. La constitution spécifie que le corps enseignant pourra bénéficier d'une réduction de la cotisation imposée aux membres, disposition qui révèle

13 Ibid.: 19. La loge n'accueillera jamais plus de 150 membres.

14 La Patrie, 10 octobre 1902; Le Journal de Montréal, 10 octobre 1902. 
bien la situation financière précaire des instituteurs et des institutrices de l'époque. Enfin, l'association compte manifestement sur le pouvoir de la presse pour propager ses idées, car elle invite, à titre gracieux, les rédacteurs de journaux à faire partie de la Ligue.

L'assemblée prend également connaissance de deux lettres d'adhésion à la Ligue de l'Enseignement: l'une provient du maire de Montréal, James Cochrane, qui applaudit à «l'esprit d'initiative et de patriotisme» des fondateurs de la Ligue; la seconde porte la signature de Joseph-Israël Tarte, propriétaire de La Patrie et ex-ministre des Travaux publics dans le ministère Laurier. «Je suis bien sûr, écrit Tarte, que vous ne songez pas à démolir, mais que votre but est d'améliorer.» Prenant la parole, Godfroy Langlois fournit cette assurance à l'assemblée et insiste sur le rôle «purement national et patriotique» qu'entend jouer la nouvelle association ${ }^{15}$.

L'intervention de Langlois n'est pas superflue. En effet, la Ligue a emprunté son nom à la Ligue française de l'Enseignement, dont le président, Ferdinand Buisson, est un franc-maçon notoire. Fondée en 1866 par Jean Macé, la Ligue française a soutenu avec enthousiasme la politique républicaine de Jules Ferry qui a établi, dans les années 1880, l'école laïque, gratuite et obligatoire. Dès le départ, l'association est liée étroitement à la franc-maçonnerie, qui la reconnaît bientôt comme sa fille aînée. La Ligue ne tarde pas à subir les attaques des conservateurs et de la hiérarchie catholique. Le Pape Pie IX la considère comme une "société pernicieuse» qui travaille à «extirper radicalement, surtout de l'âme des enfants, la foi catholique» ${ }^{16}$. Au début du XXe siècle, les auteurs catholiques verront en elle une ardente collaboratrice du gouvernement anticlérical d'Émile Combes ${ }^{17}$.

Godfroy Langlois n'ignore certes pas l'existence de la Ligue française. À l'assemblée du 9 octobre, il a brièvement fait allusion à cette dernière et à l'influence considérable qu'elle exerce dans le secteur éducatif ${ }^{18}$. L'idée même de nommer l'association québécoise d'après la Ligue française a été suggérée à Langlois par un autre libéral avancé, l'avocat Honoré Gervais, qui a également assisté à la séance du 9 octobre ${ }^{19}$. Les promoteurs de la Ligue québécoise manifestent néanmoins le désir de détruire toute équivoque

15 Voir La Patrie, 21 novembre 1902, pour le compte rendu de la séance.

16 Cité dans Mona Ouzouf, L'École, l'Église et la République (Paris, 1963), 375-376. Voir aussi Pierre Chevallier, Histoire de la franc-maçonnerie française (Paris, 1975), 3: 35 .

17 Voir par exemple, Michel Le François, Le plan maçonnique (Lille, 1905) et Georges Goyau, L'École d'aujourd'hui (Paris, 1899-1906), 2 vols.

18 Le Journal de Montréal, 10 octobre 1902.

19 Robert Rumilly, op. cit., 11: 10. 
concernant la nouvelle association. Celle-ci, affirme Arthur Beauchesne dans Le Journal, "ouvre ses portes à tous les citoyens qui désirent perfectionner notre système d'éducation. Le radical ou l'ultramontain peut en faire partie; le conservateur peut y coudoyer le libéral. C'est certainement le meilleur moyen de dégager de l'influence des partis la cause sacrée de l'éducation. ${ }^{20}$

Un fascicule publié par la Ligue quelque temps après sa fondation $^{21}$ trace, à cet égard, un portrait assez révélateur des principaux sympathisants de la réforme scolaire au début du siècle. Elle contient, en effet, le nom et la profession des membres de l'association. On y retrouve d'abord le personnel du Bureau de direction. Aux officiers élus le 9 octobre se sont rajoutés un second vice-président, le Dr Louis Laberge, directeur du Bureau d'hygiène de Montréal, et huit conseillers. Parmi ces derniers, on retrouve l'échevin Paul-G. Martineau, un libéral avancé qui s'est prononcé jadis pour l'école gratuite et obligatoire ${ }^{22}$. Siégeant également à la Commission des Ecoles catholiques de Montréal, Martineau s'affirme actuellement comme le plus chaud partisan d'une série de réformes inspirées, pour la plupart, de la pratique américaine: inspection médicale des écoles, création de bibliothèques et de gymnases, développement de l'enseignement manuel, et surtout, gratuité et uniformité des manuels scolaires.

Près de deux cents noms apparaissent ensuite sur la liste des membres. On se rend vite compte que la Ligue a recruté à Montréal la majorité de ses effectifs, et que ces derniers appartiennent pour la plupart à la petite et moyenne bourgeoisie francophone. La liste ne renferme par contre aucun ecclésiastique. De plus, les travailleurs n'y figurent qu'en très petit nombre. On ne reconnaît qu'un seul chef ouvrier, le socialiste Albert Saint-Martin, qui ne sera d'ailleurs pas actif au sein de la Ligue $^{23}$. Plus frappante encore est la place marginale occupée par les enseignants: cinq à peine ont adhéré à la Ligue. Les professions libérales sont pour leur part fortement représentées, en particulier les avocats et les notaires qui dominent le groupe des médecins. Une quinzaine de marchands se sont également inscrits, de même qu'un certain nombre de journalistes, d'ingénieurs et d'architectes. Enfin, les fonctionnaires publics - tant fédéraux, provinciaux que municipaux - forment un contingent de taille. Signalons, au niveau provincial, l'adhésion du docteur E.-P. Lachapelle, président du Bureau d'hygiène de la province de Québec, et de O.-E. Dalaire un confé-

20 Le Journal de Montréal, 21 janvier 1903.

21 La Ligue de l'Enseignement, La Ligue de l'Enseignement (Montréal, 1902).

22 Voir La Vérité, 4 mars 1882. réal, 1977), 32.

23 Claude Larivière, Albert Saint-Martin, militant d'avant-garde (Laval, Mont- 
rencier agricole très actif attaché au ministère québécois de l'Agriculture. Il faut aussi relever dans ce groupe la présence d'Errol Bouchette, un fervent nationaliste qui, dans un ouvrage très remarqué publié en 1901, Emparons-nous de l'industrie, a invité ses compatriotes à participer autrement qu'en spectateurs au développement économique du Québec.

Toutefois, c'est au sein des milieux politiques que la Ligue de l'Enseignement a recruté ses principales têtes d'affiche. En effet, ses premiers rangs sont occupés par cinq sénateurs, trois conseillers législatifs, deux ex-ministres et deux membres du cabinet Parent, soit le procureur général, Horace Archambault, et le ministre de l'Agriculture, Adélard Turgeon. Quatre juges et onze députés, dont le président de la Chambre des Communes, L.-P. Brodeur, et le président de l'Assemblée législative, H.-B. Rainville, apparaissent également en tête de liste. Le maire de Montréal, James Cochrane, et quatre échevins représentent, pour leur part, la scène municipale.

Ces hommes politiques appartiennent massivement au parti libéral, bien que l'on repère ici et là quelques figures conservatrices. De plus, un grand nombre d'entre eux mènent parallèlement une carrière dans la politique et dans les affaires. C'est ainsi que la Ligue a obtenu l'adhésion des entrepreneurs francophones les plus influents de cette période: le financier et spéculateur Louis-Joseph Forget, l'avocat-administrateur Frédéric-Liguori Béïque et l'industriel Damien Rolland. À ce trio prestigieux s'ajoutent plusieurs représentants de la moyenne bourgeoisie montréalaise. Mentionnons, entre autres, Hormisdas Laporte, échevin et négociant en gros, l'agent financier Gaspard de Serres et le président de la Banque provinciale, Guillaume-Narcisse Ducharme.

On retrouve, chez ce groupe, des membres anciens et actuels de la Commission des Écoles catholiques de Montréal: H.-B. Rainville, F.-L. Béique et Philippe Demers, député de Saint-Jean ${ }^{24}$. Ce même groupe exerce aussi un rôle prépondérant dans des organisations civiques et philanthropiques. L'une des plus actives est la Société Saint-Jean-Baptiste de Montréal, présidée depuis 1899 par le sénateur Béique, auquel succédera Hormisdas Laporte en 1905. En fait, un relevé des principaux officiers élus par la Société au début du XXe siècle démontre que ces derniers ont adhéré en grand nombre à la Ligue ${ }^{25}$. À cette époque, les dirigeants de la ${ }^{24}$ H.-B. Rainville a siégé de 1883 à 1886, F.-L. Béïque, de 1891 à 1894. L.-P. Demers siégera de 1899 à 1904 .

${ }_{25}$ Nous avons effectué ce relevé à l'aide de l'ouvrage de Robert Rumilly, Histoire de la Société Saint-Jean-Baptiste de Montréal (Montréal, 1975). Parmi les chefs de file de la Société qui ont adhéré à la Ligue, mentionnons, outre F.-L. Béique et H. Laporte, Damien Rolland, Gaspard de Serres, le sénateur Raoul Dandurand, G.-N. Ducharme, le marchand J.-M. Wilson, le docteur E.-P. Lachapelle et L.-O. David, greffier de la Ville de Montréal. 
Société sont clairement conscients que le Québec est entré dans l'âge industriel et qu'il faut, par conséquent, adapter le système éducatif aux besoins nouveaux de l'économie. C'est pourquoi ils sont parmi les premiers à réclamer le développement de l'enseignement technique, encore dans un état embryonnaire au Québec. La Société pose elle-même un geste important dans ce sens en organisant des cours publics gratuits portant sur des matières telles que la mécanique appliquée, la métallurgie et l'architecture ${ }^{26}$. De concert avec la Chambre de Commerce de Montréal, elle revendique également la création d'une École des Hautes Études commerciales, grâce à laquelle se formeraient des hommes d'affaires et des administrateurs capables d'assumer des postes de commande ${ }^{27}$.

Bref, la Ligue de l'Enseignement recrute à ses débuts une forte majorité de bourgeois libéraux intéressés aux questions éducatives, dont l'influence n'est certes pas négligeable. L'élément radical gravitant autour de la loge L'Émancipation y est bien représenté par son principal porte-parole, Godfroy Langlois ${ }^{28}$. La Ligue véhiculera évidemment plusieurs de ses idées dans le domaine scolaire. Mais, comme nous le verrons maintenant, la Ligue et ses animateurs expriment aussi des préoccupations qui dépassent les visées laïcisantes des radicaux.

\section{III - La Ligue et la réforme du système éducatif québécois}

C'est par le biais de la presse à grand tirage que la Ligue et ses promoteurs feront campagne pour une réforme du système scolaire québécois. Ils utiliseront d'abord les colonnes de La Patrie, organe des libéraux à Montréal. Ce choix est tout naturel: Godfroy Langlois est rédacteur du journal qui, par ailleurs, a pour propriétaire Joseph-Israël Tarte, l'un des premiers hommes publics à joindre la nouvelle association. Le journal relate avec éclat la fondation de la Ligue et rend compte des nouvelles inscriptions enregistrées par l'association. De plus, La Patrie entreprend, en septembre 1902, la publication d'une chronique scolaire intitulée «Autour de l'école», que rédigeront Langlois et deux autres membres de la Ligue, Napoléon Brisebois et Bernard Lippens ${ }^{29}$. "Nous invitons, écrivent les auteurs de la chronique, tous ceux qui dans le clergé, les professions libérales, le commerce et le public en général s'inté-

26 Ibid., 172.

27 Ibid., 182.

28 En 1910, dans un opuscule intitulé La Loge L'Émancipation (Montréal, 1910), un membre de l'Association catholique de la jeunesse canadienne-française, Albert Lemieux, livre au public les noms des principaux membres de la loge. Parmi ces derniers, on retrouvera ces anciens membres de la Ligue de l'Enseignement: Godfroy Langlois, le docteur Louis Laberge, le docteur Adelstan de Martigny et l'avocat Gonzalve Desaulniers.

29 La Patrie, 5 septembre 1902. 
ressent à la question scolaire à s'enrôler dans la Ligue.»" ${ }^{30}$ À la suite d'une querelle interne au sein du parti libéral, Langlois quitte La Patrie au début de 1903 pour devenir en avril de la même année, le directeur-gérant d'un nouveau journal, Le Canada, qui remplace La Patrie comme organe officiel du parti libéral dans la métropole. L'instigateur de cette fondation est nul autre que le sénateur Béïque. Le président de la Ligue, Olivier Faucher, et d'autres membres de l'association, tels H.-B. Rainville et le sénateur Raoul Dandurand, figurent aussi parmi les actionnaires et les administrateurs du Canada ${ }^{31}$. La Ligue pourra ainsi compter sur le journal pour exposer son point de vue au public et, en particulier, pour récuser les attaques violentes dont elle sera bientôt la cible.

C'est donc en puisant dans La Patrie et Le Canada qu'il est possible de retracer les principaux éléments de la campagne menée par la Ligue et ses animateurs, depuis sa fondation jusqu'à sa mise en veilleuse en 1904. En 1903, Godfroy Langlois et Arthur Beauchesne rédigent également une brochure sur La Question de l'Instruction publique dans la province de Québec. La préface qui se présente sous la forme d'un manifeste, renferme une déclaration de principes bien articulée. La brochure explore ensuite plus en détail plusieurs des thèmes déjà abordés dans les deux journaux mentionnés ci-dessus. Pour les fins de notre démonstration, nous avons partagé ces thèmes en deux champs d'analyse principaux. Nous avons d'abord réuni ceux qui traitent de questions reliées directement à la spécificité de l'organisation scolaire au Québec, c'est-à-dire le rôle de l'Etat dans le secteur éducatif, l'administration du système scolaire public et la formation des maîtres. Nous avons regroupé ensuite les thèmes qui traduisent des préoccupations se rattachant au nouveau contexte socio-économique de la période, soit l'organisation pédagogique de l'enseignement primaire, la centralisation des écoles rurales, l'enseignement technique et l'éducation des filles. Nous débuterons toutefois notre analyse en définissant la conception et les objectifs fondamentaux que la Ligue de l'Enseignement assigne au processus éducatif.

\section{a) Conception et objectifs de l'éducation}

L'essentiel des réformes proposées par la Ligue de l'Enseignement est centré sur l'enseignement primaire, que l'association considère comme le pilier majeur de l'édifice scolaire. Cette conviction repose sur un postulat exprimé en ces termes: «l'instruction pri-

30 Ibid., 29 novembre 1902, Chronique: «Autour de l'école»; Robert Rumilly, op. cit., 11: 11 .

31 André Beaulieu et Jean Hamelin, La presse québécoise des origines à nos jours (Québec, 1979), 4 (1896-1910): 166. 
maire est devenu un droit dont personne ne doit être privé.»» ${ }^{32} \mathrm{Ce}$ principe de «l'instruction pour tous», souligne la Ligue, s'harmonise «avec les principes de notre forme de gouvernement, qui reconnaît la liberté individuelle, l'égalité des droits civils et l'élection populaire» ${ }^{33}$. L'école primaire constitue donc l'école de tout le peuple et elle a pour but «de donner à tous, pauvres et riches, les connaissances devenues indispensables à la vie» ${ }^{34}$. L'association assigne ainsi un objectif utilitaire à l'enseignement primaire. En plus d'apprendre «leurs devoirs envers Dieu, envers leur prochain et envers eux-mêmes», les jeunes Québécois doivent, en effet, acquérir un degré de savoir qui leur sera utile «dans n'importe quelle condition de la vie» ${ }^{35}$. La Ligue éprouve à cet égard une admiration visible pour le système scolaire des États-Unis qui fournit une "éducation solide et pratique» à tous les élèves américains ${ }^{36}$. Malheureusement, tel n'est pas le cas dans la province de Québec, où «des milliers et des milliers de nos compatriotes gagnent leur vie à la journée, dans les manufactures, dans des travaux plus pénibles encore qui, avec une instruction plus pratique, eussent pu se frayer un chemin dans la vie» ${ }^{37}$.

Mais la Ligue attribue à l'instruction un rôle encore plus ambitieux. Elle lui apparaît, en effet, comme une condition indispensable à l'émancipation économique collective des Canadiens français. La Ligue soutient ainsi que

dans l'état actuel de la société, en présence de la lutte que se font les peuples sur le terrain du commerce et de l'industrie, de l'agriculture, de l'influence politique, l'instruction populaire est devenue un facteur tellement important qu'aucun pays ne peut le négliger sans se laisser devancer par ses rivaux. ${ }^{38}$

Bref, il y va de l'intérêt national d'améliorer le système scolaire public puisque, affirme la Ligue, «c'est par le relèvement de l'école primaire qu'il [le peuple canadien-français] s'assurera la suprématie à laquelle il a le droit d'aspirer dans ce grand pays, qu'il conservera son indépendance, sa langue, sa tradition et ses droits» ${ }^{39}$.

Tout en faisant valoir l'urgence de répandre au plus tôt l'instruction publique, la Ligue envisage l'avenir avec optimisme. Cette

32 La Question de l'instruction publique (Montréal, 1903), ii.

33 Ibid., 1.

34 Ibid., 1.

35 Ibid., 1.

36 "Lowell», éditorial, La Patrie, 10 janvier 1903; «L'instruction publique aux États-Unis», éditorial, ibid., 17 janvier 1903. Le Canada, 14 mai 1903.

37 La Question..., op. cit., i.

38 La Patrie, 21 novembre 1902.

39 La Question, op. cit., 1. 
attitude résulte de la confiance inébranlable qu'elle place dans le processus éducatif. Avec l'école, tout lui semble possible, puisque les Canadiens français possèdent au départ les aptitudes physiques et intellectuelles nécessaires ${ }^{40}$. Une telle estimation du rôle de l'école empêche malheureusement la Ligue de peser avec réalisme les rapports de force existant entre la collectivité francophone et la minorité anglo-québécoise qui détient les leviers de commande dans le domaine économique.

\section{b) Le rôle de l'État dans le secteur éducatif}

Dès les premières lignes de la brochure qu'elle publie en 1903, la Ligue déclare que «l'instruction publique est une charge d'État» ${ }^{41}$. Cette affirmation du rôle positif de l'État en matière d'éducation reflète fondamentalement les convictions de Godfroy Langlois et des libéraux radicaux. Langlois ne dissimule pas son admiration pour la France et les autres nations «civilisées» qui ont constitué l'enseignement primaire en un service public dirigé entièrement par le pouvoir civil ${ }^{42}$. Cette conception de l'enseignement primaire comme service public s'appuie sur deux idées solidaires, auxquelles souscrit la Ligue: l'égalité des enfants, riches ou pauvres, à l'école publique, et leur droit à l'instruction primaire. Au niveau des politiques éducatives, cette même conception implique deux principes essentiels: la gratuité de l'enseignement et l'obligation scolaire. Condamnés explicitement par l'Église, ces principes n'ont pas encore été appliqués au Québec. La Ligue les approuve cependant, quoique de façon discrète et détournée. La chronique scolaire de La Patrie réclame ainsi l'uniformité et la gratuité des manuels scolaires, première étape vers la gratuité totale ${ }^{43}$. Paul-G. Martineau soumet lui-même cette requête à ses collègues de la Commission des Écoles catholiques de Montréal ${ }^{44}$. La Patrie fait aussi état de la campagne que mène, à la fin de 1902, le Star de Montréal en faveur de l'instruction gratuite au Québec ${ }^{45}$. Enfin, le journal ne craint pas de citer en exemple les États américains voisins qui, tel le Massachusetts, ont amélioré la fréquentation scolaire des enfants en adoptant une loi à cet effet ${ }^{46}$.

\footnotetext{
40 Ibid. «Instruisons-nous», éditorial, La Patrie, 4 novembre 1902.

41 La Question..., op. cit., i.

42 Voir, par exemple, "Instituteurs allemands et instituteurs français», Le Canada, 26 septembre 1903; «Au pays du Rhin», ibid., 17 octobre 1903.

43 "Autour de l'école», La Patrie, 17 janvier 1903, 24 janvier 1903.

44 Le Canada, 25 novembre 1903, 13 janvier 1904, 12 mars 1904.

45 Voir, par exemple, la chronique «Autour de l'école», La Patrie, 22 novembre 1902, 29 novembre 1902.

46 «L'instruction publique au Massachusetts», éditorial, La Patrie, 31 décembre 1902; «L'instruction publique aux États-Unis», éditorial, ibid., 17 janvier 1903.
} 
Toutefois, les porte-parole de la Ligue évitent de se prononcer sur les modalités d'application concrètes de la gratuité et de l'obligation scolaires. Ils sont aussi silencieux en ce qui concerne les structures supérieures du système éducatif. En effet, même si le principe de «l'État éducateur» est énoncé, il n'est question, ni de l'abolition ou de la réforme du Conseil de l'Instruction publique, ni du projet de rétablir un ministère responsable. Quant à ce fonctionnaire inamovible, le surintendant de l'Instruction publique, on déplore, sans plus, sa mollesse et son manque d'initiative ${ }^{47}$. Bref, tout en revendiquant une action plus agressive de l'État, la Ligue n'explique pas comment celle-ci pourra s'exercer dans le cadre des institutions en place. La discussion se maintient au niveau des idées, les solutions empiriques faisant ici nettement défaut. La Ligue a conscience de s'engager sur un terrain dangereux, d'où le caractère timide et hésitant de son intervention.

\section{c) L'administration du système scolaire public}

Sans dénoncer ouvertement les structures éducatives existantes, la Ligue de l'Enseignement désire tout de même l'édification d'un système scolaire uniforme et centralisé, tel qu'il s'est constitué en Ontario et dans plusieurs États américains. Depuis le milieu du XIXe siècle, le développement de l'organisation scolaire au Québec a obéi fidèlement au principe d'une décentralisation poussée. Au début du XXe siècle, les milieux éducatifs catholiques n'ont pas encore livré ou soutenu une lutte visant à renverser ce mouvement. La loi de l'Instruction publique confère toujours aux commissions scolaires locales une large autonomie et des responsabilités très étendues, comprenant la construction et l'entretien des écoles, la rétribution du personnel enseignant, l'application du programme d'études et la perception des contributions scolaires ${ }^{48}$.

La Ligue considère cette délégation de pouvoirs comme une source constante d'abus et d'inefficacité. Elle s'élève vivement contre la piètre performance des commissaires d'écoles qui négligent grossièrement leurs devoirs, surtout en ce qui concerne l'amélioration des bâtiments scolaires et la rémunération des enseignants $^{49}$. L'association fait sienne la cause des instituteurs et des

47 «Autour de l'école», La Patrie, 13 septembre 1902, 17 janvier 1903.

48 Paul de Cazes, L'Instruction publique dans la province de Québec (Québec, 1905), 22-26. C.-J. Magnan, directeur de la revue pédagogique, $L$ 'Enseignement primaire, observe justement à cette époque que "chaque commission constitue en quelque sorte un petit gouvernement, pratiquement indépendant du pouvoir central». L'Enseignement primaire (juin 1903): 579.

${ }_{49}$ La Question de l'instruction publique, op. cit., 14-15. «Nos maisons d'école», éditorial, La Patrie, 27 novembre 1902; "Autour de l'école», ibid., 10 janvier 1903. Voir aussi Le Canada, 23 juin 1903, 29 mars 1904. 
institutrices, à qui les commissaires accordent des salaires dérisoires et souvent insuffisants pour assurer leur subsistance. La mesquinerie des commissaires se manifeste avant tout à l'endroit des jeunes institutrices, dont plusieurs reçoivent dans les comtés ruraux un traitement annuel inférieur à $100.00 \$ 50$. Afin de sensibiliser l'opinion publique au triste sort des institutrices, La Patrie leur ouvre les colonnes de sa chronique scolaire. Les institutrices répondent massivement à l'appel. Leurs lettres dénoncent à l'unanimité la conduite malveillante des commissaires d'écoles, qui est dictée tour à tour par l'incompétence, le jeu du patronage et l'ambition ultime d'épargner quelques dollars. La correspondance révèle aussi leur ignorance - consciente ou involontaire - de la loi scolaire. La plupart, par exemple, enfreignent les règlements du Comité catholique en obligeant les maîtresses à défrayer, à même leurs maigres émoluments, le coût du bois ou du charbon nécessaire au chauffage de l'école ${ }^{51}$.

Une solution au problème serait que le gouvernement fixe des normes salariales pour le corps enseignant, ce qui protégerait ce dernier des pressions locales. La Ligue hésite à réclamer directement le statut de "fonctionnaires de l'État» pour les instituteurs et les institutrices, mais elle appuie les partisans d'une telle mesure ${ }^{52}$. Ce changement exigerait en retour une révision fondamentale de la politique budgétaire du gouvernement provincial, qui impose aux commissions scolaires le fardeau financier de l'enseignement primaire public. Or, la Ligue dénonce à cet égard l'État québécois qui, soutient l'association, a gravement ignoré ses responsabilités au niveau du financement de l'éducation ${ }^{53}$. La Patrie abonde dans ce sens et expose, chiffres à l'appui, la position «humiliante» du Québec, qui serait au dernier rang de la Confédération au chapitre des octrois réservés à l'instruction publique ${ }^{54}$. Le journal va jusqu'à reproduire un article diffamatoire de l'Ottawa Journal, lequel qualifie de "disgrâce nationale» le budget scolaire du gouvernement québécois ${ }^{55}$.

Selon la Ligue de l'Enseignement, l'accroissement des subsides gouvernementaux est d'autant plus vital qu'il entraînera un

50 La Question de l'instruction publique, op. cit., 32-33. Voir aussi «Une situation désespérée», éditorial, La Patrie, 12 janvier 1903.

51 Parmi les lettres les plus intéressantes adressées par les institutrices à La Patrie, mentionnons celles publiées les 20 septembre, 4 octobre, 8 novembre et 6 décembre 1902 dans la chronique «Autour de l'école». Sur cette question, voir aussi «Plaintes et projets des institutrices», Le Canada, 28 août 1903.

52 Voir La Question de l'instruction publique, op. cit., 35. Voir aussi «Autour de l'école», La Patrie, 4 octobre 1902.

${ }_{53}$ La Question..., op. cit., 8-9.

54 «Ce que nous dépensons pour l'instruction publique», éditorial, La Patrie, 22 octobre 1902; «Le relèvement de l'école s'impose», éditorial, ibid., 18 novembre 1902.

55 «A National Disgrace», éditorial, La Patrie, 16 janvier 1903. 
renforcement de l'autorité centrale sur les pouvoirs locaux. Les commissions scolaires hésiteront, en effet, à ignorer la loi et les règlements provinciaux de crainte qu'on ne leur retire un octroi substantiel. Présentement, c'est la situation inverse qui prévaut, les commissaires préférant agir à leur guise, au risque de se priver d'une allocation qu'ils jugent insignifiante. Ainsi, observe la Ligue, «notre loi scolaire n'atteint pas le but désiré, parce que ceux qui sont chargés de son exécution n'ont pas un levier assez puissant pour faire agir les justiciables auxquels incombe la tâche de l'observer» ${ }^{56}$.

La centralisation à laquelle aspire la Ligue de l'Enseignement s'accomplira également grâce à deux réformes importantes. Il faut d'abord améliorer et étendre le système d'inspection scolaire. L'augmentation du nombre des inspecteurs, un salaire équivalent à l'importance de leurs fonctions et, surtout, la nomination d'un inspecteur provincial permettront d'atteindre cet objectif ${ }^{57}$. En second lieu, il importe d'obtenir une application uniforme du programme d'études. L'instauration d'examens provinciaux est perçue à cet égard comme une formule centralisatrice efficace puisqu'elle amènera les maîtres et les élèves à se conformer aux prescriptions du programme officiel. Godfroy Langlois endosse ainsi dans Le Canada l'Association des institutrices catholiques de Québec qui réclame à l'époque la création de tels examens. Ceux-ci seraient préparés directement par le Département de l'Instruction publique, qui distribuerait en retour des «certificats de promotion» aux élèves ayant réussi les épreuves ${ }^{58}$.

\section{d) La formation des maîtres}

L'unification de toute organisation scolaire implique la formation uniforme des maîtres. Au début du XXe siècle, l'homogénéité du personnel enseignant catholique n'a pas encore été réalisée au Québec. En effet, la loi scolaire n'oblige pas les futurs instituteurs et institutrices à fréquenter les Écoles normales. Bien au contraire, la majorité d'entre eux se contentent du brevet de capacité accordé sur simple examen par le Bureau central des examinateurs catholiques, établi en 1898 . Ces brevets sont distribués avec une grande indulgence; ils ont de plus la même valeur que les diplômes décernés par les Écoles normales après un stage d'études théoriques et pratiques. Il existe donc au Québec deux systèmes de certification dont les exigences sont fort différentes. De plus, une loi scolaire adoptée en 1846 dispense les enseignants religieux du bre-

\footnotetext{
56 La Question..., op. cit., 12.

57 "Autour de l'école», La Patrie, 4 octobre, 29 novembre, 6 décembre 1902.

58 «Plaintes et projets des institutrices», Le Canada, 28 août 1903.
} 
vet de capacité. Le statut spécial conféré à ce groupe prend toute son importance lorsqu'on considère qu'en 1900, les Frères et les Soeurs comptent pour $44 \%$ du personnel enseignant au Québec ${ }^{59}$.

La Ligue de l'Enseignement ne se prononce pas sur la cléricalisation des effectifs enseignants, ni sur l'exemption dont jouissent les religieux, question pourtant vivement débattue par les libéraux radicaux à la fin du XIXe siècle ${ }^{60}$. Elle dirige plutôt son tir contre le Bureau central dont elle réclame l'abolition immédiate. Cette «fabrique à diplômes», souligne l'association, affecte négativement la carrière enseignante. Elle provoque une concurrence funeste aux finissants normaliens, crée un surplus d'enseignants aux qualifications douteuses, surplus qui, à son tour, maintient l'insuffisance des salaires ${ }^{61}$. Enfin, en permettant aux jeunes filles de se présenter à l'examen dès l'âge de 16 ans, le Bureau encourage les commissions scolaires à violer la loi qui fixe à 18 ans l'âge minimum exigé pour enseigner. Le résultat final est que les institutrices de mérite sont mises «dans l'alternative d'accepter un traitement qu'une servante refuserait avec dédain ou de céder la place à des débutantes (...) qui s'offrent à vil prix et font aux dépens des élèves et du public l'apprentissage qu'elles auraient dû faire avant de prendre la direction d'une école» ${ }^{62}$.

L'essentiel, estime la Ligue, est de viser à la qualité et non à la quantité. C'est pourquoi les Écoles normales doivent constituer la seule voie d'accès à la carrière enseignante. Et comme ces institutions sont très peu nombreuses dans la province, il convient d'en fonder de nouvelles sans tarder. La Ligue préconise d'abord la fondation d'Écoles normales pour filles, puisque ce sont ces dernières qui envahissent le secteur primaire ${ }^{63}$. Même si elle préférerait voir des maîtres enseigner aux garçons - surtout aux adolescents - , la Ligue semble accepter comme un fait accompli la féminisation du personnel enseignant. La réponse logique à ce phénomène, raisonne-t-elle, n'est pas de chercher à le renverser, mais plutôt de pourvoir au plus tôt à la préparation d'institutrices qualifiées ${ }^{64}$. tableau 6.

59 Louis-Philippe Audet, Histoire de l'enseignement au Québec, op. cit., 2: 317,

${ }_{60}$ R. Heap, op. cit., 361-390.

61 "Autour de l'école», La Patrie, 13 septembre, 11 octobre, 18 octobre, 31 octobre, 27 décembre 1902. Voir aussi «Dans un état de démoralisation», éditorial, ibid., 16 décembre 1902. bre 1902.

62 La Question..., op. cit., 19. Voir aussi «Autour de l'école», La Patrie, 4 octo-

63 "Autour de l'école», La Patrie, 25 octobre 1902, 13 décembre 1902, 17 janvier 1903. Voir aussi «Brevets d'écoles normales», éditorial, ibid., 22 novembre 1922; «Dans un état de démoralisation», éditorial, ibid., 16 décembre 1902.

64 Voir La Question de l'Instruction publique, op. cit., 21-23. 


\section{IV - La Ligue et l'«Éducation nouvelle»}

En plus de vouloir apporter des correctifs à l'organisation scolaire du Québec, les animateurs de la Ligue de l'Enseignement réclament une adaptation du système éducatif aux conditions nouvelles créées par l'industrialisation et l'urbanisation. Les mesures qu'ils préconisent à cet égard s'inscrivent dans un vaste courant réformiste occidental qui revendique à l'époque une «éducation nouvelle» destinée à intégrer harmonieusement les enfants à la société moderne tout en les protégeant de ses écueils. À l'instar des promoteurs de l'«éducation nouvelle», la Ligue préconise en effet une série de réformes visant, d'une part, à renouveler les conceptions et les méthodes pédagogiques, et, d'autre part, à imprimer un caractère utilitaire à l'école en reliant cette dernière au milieu dans lequel elle évolue et en axant davantage les cours d'études sur l'orientation future des élèves. Cette dimension du programme de la Ligue que nous examinerons maintenant n'a donc rien d'original; elle révèle au contraire l'apport des influences extérieures dans l'élaboration de la pensée réformiste québécoise au début du siècle.

\section{a) L'organisation pédagogique des écoles primaires}

\section{Conceptions et méthodes pédagogiques}

Comme un grand nombre de réformistes sociaux de l'époque, les animateurs de la Ligue manifestent pour l'enfant un intérêt empreint de sympathie et de considération. Dans le domaine de l'éducation, ce sentiment se traduit par une attitude qui envisage l'enfant comme un être différent et original. Il faut donc que l'école primaire assure l'épanouissement de l'élève en respectant ses goûts, ses aptitudes et ses besoins spécifiques. C'est pourquoi la Ligue préconise des mesures propres à ajuster l'enseignement primaire au profil intellectuel et psychologique de l'enfant.

La Ligue demande d'abord l'établissement de classes graduées qui regrouperaient chacune les élèves du même âge et du même degré. À l'époque, la majorité des écoles de la province sont dirigées par une seule institutrice qui se voit confier des classes d'enfants de tout âge, qu'elle subdivise alors en sections correspondant aux diverses années du cours primaire. Et comme elle ne peut s'occuper que d'une division à la fois, l'institutrice est obligée de soumettre les élèves à un régime disciplinaire «mortellement ennuyant et en opposition avec leur nature et leurs besoins» ${ }^{65}$.

La Ligue voudrait aussi que la loi scolaire interdise formellement l'accès à l'école primaire aux enfants de 5 à 7 ans. Elle s'ob-

${ }^{65}$ Ibid., 28. 
jecte, en effet, à ce que l'on contraigne des intelligences aussi jeunes à assimiler les matières régulières du cours d'études. Il faut, au contraire, créer à leur intention une institution spécifique, soit l'école maternelle ou le jardin d'enfance, où sera pratiquée une pédagogie «en harmonie avec l'âge, les besoins réels, les forces physiques et morales 'tout petits' $\gg{ }^{66}$. L'enseignement donné à l'école maternelle s'inspirera alors des thèses pédagogiques développées par Froebel et auxquelles souscrivent les tenants de l'«éducation nouvelle». Ainsi, il cultivera l'élan vital et la spontanéité des enfants en accordant un rôle essentiel à l'activité physique et aux jeux récréatifs ${ }^{67}$.

Enfin, la Ligue réclame un renouvellement des méthodes pédagogiques pratiquées à l'école primaire. Elle reproche aux enseignants de recourir trop exclusivement à la mémorisation et à la récitation littérale passive ${ }^{68}$. Ces derniers doivent adopter plutôt la démarche intuitive élaborée par l'éducateur suisse Pestalozzi. Il s'agit pour le maître de passer de l'objet à l'idée, du concret à l'abstrait. La leçon de choses, exercice d'observation par excellence, constitue le pivot central de cette méthode qui s'adresse aux sens de l'enfant et le soustrait au dogmatisme des mots ${ }^{69}$.

\section{Le programme d'études}

La Ligue et ses promoteurs sont conscients que l'industrialisation et l'urbanisation imposent à l'école des fonctions et des responsabilités nouvelles qui exigent une révision du programme d'études. Fondée à Montréal, la Ligue est ouverte aux effets pernicieux d'une croissance urbaine rapide sur les conditions de vie. Elle se soucie particulièrement de la santé et du bien-être physique des jeunes citadins. C'est pourquoi elle demande un enseignement accru de l'hygiène à l'école primaire ${ }^{70}$. L'association exprime surtout ici le point de vue de deux de ses membres, le docteur Laberge et le docteur Lachapelle, qui multiplient à l'époque les efforts afin d'améliorer la qualité de l'hygiène dans la métropole ${ }^{71}$. Inspiré par l'exemple des écoles urbaines américaines, Paul-G. Martineau espère, pour sa part, amener la Commission des Écoles catholiques de Montréal à établir des gymnases où seraient dispensés des cours de culture physique aux élèves ${ }^{72}$.

66 Ibid.

67 Ibid., 30-31.

68 «Autour de l'école», La Patrie, 20 septembre 1902, 27 septembre 1902.

69 "Autour de l'école», La Patrie, 18 octobre 1902.

70 "Hygiène et santé publique», La Patrie, 6 décembre 1902; «Autour de l'école», ibid., 3 janvier 1903; "Questions d'hygiène», Le Canada, 18 février 1904.

71 "Cours gratuits d'hygiène», La Patrie, 14 novembre 1902. "A l'hygiène», Le Canada, 4 avril 1903.

72 «Les écoles catholiques», Le Canada, 17 décembre 1903. 
Malgré ses racines urbaines, la Ligue se préoccupe tout autant de l'enseignement qui se donne dans les écoles rurales. Ses porteparole envisagent avec appréhension la dépopulation croissante des campagnes. Ils s'inquiètent surtout de l'exode des jeunes ruraux qui désertent le métier de cultivateur pour s'orienter vers le commerce, l'industrie et les professions libérales. Un membre de la Ligue, le conférencier agricole O.-E. Dalaire, soutient que l'école primaire rurale peut contrecarrer ce mouvement si on lui assigne un programme d'études adapté aux besoins spécifiques de la population agricole ${ }^{73}$. C'est pourquoi il insiste sur la nécessité de développer et de fortifier l'enseignement de l'agriculture, auquel on a, jusqu'à ce jour, accordé très peu d'importance ${ }^{74}$. Afin de relier l'école primaire rurale à son milieu, la Ligue propose également la création de jardins scolaires ruraux, où les élèves s'adonneront à l'étude pratique de la botanique et de l'horticulture. Largement répandu en France, en Belgique et en Suède, ce mode d'enseignement initiera les jeunes ruraux au travail agricole et permettra l'application des nouvelles méthodes pédagogiques centrées sur une participation active de l'enfant ${ }^{75}$.

Selon O.-E. Dalaire, le but fondamental de l'enseignement agricole est «qu'au sortir de l'école, les enfants ne rougissent pas de la profession de leur père, qu'ils s'attachent au contraire au sol de la patrie» ${ }^{76}$. De même, La Patrie considère que "la tâche et l'effort constant de l'instituteur et de l'institutrice devraient être de pénétrer les fils des paysans des beautés de la vie rurale, de la liberté qu'elle offre, de la santé qu'elle procure, du bonheur qu'elle assure» ${ }^{77}$. Si le journal ne condamne pas directement le milieu urbain, il n'hésite pas à contraster les vicissitudes de la vie ouvrière avec l'existence sereine de l'agriculteur ${ }^{78}$. Un enseignement "ruralisé»" devra donc exposer les avantages de l'agriculture, non seulement comme activité économique, mais aussi comme mode de vie. Les porte-parole de la Ligue véhiculent ici une pensée agrarienne qui emprunte beaucoup au credo agriculturiste prêché à l'époque par les milieux cléricaux et conservateurs. Toutefois, on ne saurait les considérer comme des agriculturistes convaincus puisqu'ils favorisent en même temps l'industrialisation et le développement économique intégral de la province de Québec.

\footnotetext{
73 «Autour de l'école», La Patrie, 18 octobre 1902.

74 "Autour de l'école», La Patrie, 31 octobre 1902, 15 novembre 1902.

75 La Question..., op. cit., 16-17. "Autour de l'école», La Patrie, 6 décembre 1902; «L'agriculture à l'école», éditorial, ibid., 7 octobre 1902.

76 Cité dans la chronique «Autour de l'école», La Patrie, 22 novembre 1902.

77 «L'agriculture à l'école», éditorial, La Patrie, 7 octobre 1902.

78 Ibid.
} 


\section{b) La centralisation des écoles rurales}

Par ailleurs, le problème de l'enseignement primaire rural amène Godfroy Langlois et La Patrie à promouvoir un mouvement de centralisation scolaire dans les campagnes. Ce mouvement impliquerait la fusion de plusieurs petites écoles de rang mal équipées en une large école centrale qui accueillerait les enfants d'une paroisse ou d'un comté grâce à l'instauration d'un système de transport gratuit. La Patrie appuie fortement les démarches effectuées au début du siècle par le commissaire de l'Agriculture du Canada, James Robertson, dans le but d'établir un système d'écoles «consolidées» dans l'Est du pays. À l'instar de Robertson, le journal voit de nombreux avantages dans la concentration scolaire rurale. Elle permettra la construction de bâtiments scolaires spacieux et munis de l'équipement nécessaire à l'enseignement de l'agriculture, des sciences naturelles, des travaux manuels et domestiques. Elle donnera lieu à l'emploi de professeurs plus qualifiés et mieux rémunérés. Des classes graduées pourront aussi être mises sur pied, ce qui contribuera au progrès intellectuel des élèves. Enfin, l'établissement d'écoles centrales améliorera la fréquentation scolaire en stimulant la vie sociale des élèves et en assurant un contrôle étroit de leur présence. Déjà, souligne La Patrie, plusieurs États américains du Centre et de l'Est, dont l'Ohio, le Kansas et le Massachusetts, ont tenté l'expérience avec grand succès. Pourquoi alors, demande le journal, la province de Québec n'enverrait-elle pas aux États-Unis une délégation qui pourrait étudier la question sur place ${ }^{79}$ ?

\section{c) L'enseignement technique}

La réforme de l'enseignement primaire constitue l'objectif principal que s'est fixé la Ligue de l'Enseignement. Toutefois, ses promoteurs demandent également le développement de l'enseignement technique au niveau du secondaire. Dans la chronique "Autour de l'école», ils soulignent avec admiration les réalisations de la Prusse dans ce domaine, nation qui possédait déjà en 1886 plus de 125 écoles ouvrières professionnelles ${ }^{80}$. Ils signalent aussi les efforts qui se font à l'époque en Ontario pour établir cet enseignement dans les différentes parties de la province. "Pouvonsnous plus longtemps nous croiser les bras?», demandent-ils à leurs lecteurs ${ }^{81}$.

79 «La centralisation scolaire», éditorial, La Patrie, 13 octobre 1902; «Les écoles rurales dans l'Ohio», ibid.

80 «Autour de l'école», La Patrie, 6 décembre 1902.

81 «Autour de l'école», ibid., 13 décembre 1902. 
La Patrie se fait en même temps la championne de l'enseignement technique dans ses pages éditoriales. Le besoin d'une maind'oeuvre spécialisée et qualifiée fait valoir la nécessité de l'organiser au Québec. "L'ouvrier qui ne possède pas une bonne instruction technique, soutient-elle, n'est pas plus utile à la société qu'à sa famille. Ce sont les industries, le commerce qui font la prospérité d'un pays! ${ }^{82}$ Le journal appuie la campagne que mènent alors l'Association des Manufacturiers Canadiens et le «Montreal Board of Trade» en faveur de l'enseignement technique ${ }^{83}$. La Patrie revendique ce type de formation pour des motifs d'ordre économique: partout où il s'est développé, il a donné lieu à un admirable essor industriel, comme en témoigne l'exemple des États-Unis, de l'Allemagne et de l'Angleterre ${ }^{84}$. Mais le journal lui attribue en plus la dimension d'une oeuvre nationale. La création d'écoles techniques ouvrira, en effet, de nouvelles avenues de promotion aux Canadiens français et libérera le champ encombré des professions libérales. Jusqu'à présent, il a fallu recruter à l'étranger des chefs d'atelier et des contremaîtres parce que les francophones n'étaient pas assez qualifiés pour accomplir autre chose que «du travail à la journée». Désormais, ces derniers pourront acquérir la compétence requise pour se tailler une place de choix dans le secteur industriel et manufacturier ${ }^{85}$.

La Patrie confère d'ailleurs un caractère d'urgence à la formation professionnelle de ses compatriotes. «Si notre race ne s'outille point sans plus de retard, prévient-elle, nous descendrons l'échelle. Nous serons les serviteurs des autres! ${ }^{86}$ Cette attitude explique pourquoi le journal presse les pouvoirs publics provinciaux et municipaux de participer avec les manufacturiers à l'établissement des écoles techniques. De cette façon, les intérêts de la population francophone seraient servis adéquatement, ce qui ne serait pas assuré si cette responsabilité était abandonnée à l'initiative privée, c'est-à-dire à l'élite montréalaise anglophone qui a déjà manifesté l'intention d'ouvrir une telle école dans la métropole. «Ce que nous voulons, déclare le journal, c'est l'instruction des foules... canadiennes-françaises, comme des foules canadiennesanglaises. Nous voulons une école dans la partie est de Montréal,

82 «Écoles industrielles», éditorial, ibid., 6 février 1903.

83 "Éducation technique», ibid., 13 novembre 1902; "L'éducation technique», ibid., 30 janvier 1903; "Notre prière sera-t-elle entendue», éditorial, ibid., 31 janvier 1903.

84 «Des écoles industrielles», éditorial, ibid., 14 janvier 1903.

85 «Écoles industrielles. Pourquoi Montréal n'en aurait-il pas une?», ibid., 10 janvier 1903; "Notre prière sera-t-elle entendue?», éditorial, ibid., 31 janvier 1903; «Écoles industrielles», éditorial, ibid., 6 février 1903. «Que fait Montréal pour l'enseignement technique?», éditorial, Le Canada, 4 décembre 1903.

86 «Notre prière sera-t-elle entendue?», éditorial, ibid., 31 janvier 1903. 
où les classes laborieuses [canadiennes-françaises] constituent le gros de la population. ${ }^{87}$

On s'aperçoit, en somme, que la confiance manifestée par les animateurs de la Ligue de l'Enseignement à l'égard de l'éducation en général s'intensifie dans le cas de l'enseignement professionnel. Un éditorial de La Patrie résume fidèlement ce point de vue:

Les Canadiens français sont intelligents, ils ont des dispositions spéciales pour la mécanique. S'ils avaient l'avantage de l'instruction technique, de cette instruction pratique que l'on donne abondamment aux foules dans d'autres pays, comme aux États-Unis, par exemple, au lieu de gagner des salaires inférieurs, ils arriveraient au sommet de leur métier, ils atteindraient les plus hautes situations. ${ }^{88}$

\section{d) L'éducation des filles}

Les membres de la Ligue de l'Enseignement accordent une attention particulière à l'éducation des filles. Ils ont conscience que les nouvelles conditions socio-économiques exercent des pressions sur l'institution familiale et sur le rôle traditionnel joué par la femme dans la société. Or, ils ne remettent nullement ce rôle en question. Bien au contraire, ils considèrent que la place naturelle de la femme est au foyer, et que l'éducation reçue par la jeune fille ne doit pas l'empêcher "de s'intéresser au soin du ménage». Il importe que celle-ci réalise que «c'est pour sa famille et non pour la galerie mondaine qu'elle doit garder le rayonnement de ses talents, de ses mérites» ${ }^{89}$. Savoir équilibrer le budget familial et confectionner les vêtements contribuera beaucoup plus à l'harmonie du foyer que des connaissances intellectuelles étendues ${ }^{90}$. D'où la nécessité d'introduire à l'école primaire des cours d'économie domestique qui, en plus de donner une formation pratique et fonctionnelle, inculqueront des «habitudes morales» aux futures ménagères afin qu'elles deviennent plus tard «les ouvrières de la joie, de la santé et du bonheur domestique» ${ }^{91}$.

Déjà, les Soeurs des Saints Noms de Jésus et de Marie, les Soeurs de Sainte-Croix, les Soeurs de Sainte-Anne et plusieurs autres communautés religieuses ont ajouté à leur programme d'études l'enseignement de la coupe et de la couture. La Patrie et Le Canada se montrent enchantés de cette décision ${ }^{92}$. Godfroy Langlois invite

87 «Écoles techniques: Quelles écoles faut-il?», éditorial, ibid., 7 février 1903.

88 «Notre prière sera-t-elle entendue?», éditorial, ibid., 31 janvier 1903.

89 «Autour de l'école», ibid., 11 octobre 1902.

90 «Autour de l'école», ibid., 13 septembre 1902, 11 octobre 1902.

91 «Autour de l'école», ibid., 13 septembre 1902.

92 "Une heureuse innovation», ibid., 12 janvier 1903. "Coupe et couture», Le Canada, 27 octobre 1903; «Enseignement plus pratique», ibid., 26 mars 1904. 
aussi, dans Le Canada, les jeunes filles à suivre les cours de coupe et de couture qui sont donnés par le Conseil des arts et manufactures. «La vraie femme doit savoir tenir l'aiguille, écrit le journal, cela occupe ses loisirs, développe son goût et la tient dans son véritable milieu. ${ }^{93}$

Les promoteurs de la Ligue estiment également que la création d'écoles ménagères s'impose sans tarder, tant à la ville que dans les milieux ruraux ${ }^{94}$. L'enseignement ménager agricole revêt une importance spéciale car il est perçu comme un autre moyen de combattre la désertion des campagnes. La Patrie souhaite qu'il se développe selon le modèle belge, qui a pour but spécifique de former «des fermières actives et intelligentes, sachant diriger la bassecour et le ménage, et seconder utilement l'homme des champs dans tous ses travaux» ${ }^{95}$. Le Canada favorise aussi pour les jeunes rurales cette forme d'éducation qui «vaut surtout mieux que l'étude dispendieuse, infructueuse et malheureusement si universelle du piano ${ }^{96}$.

Tel que défini ci-dessus, l'enseignement ménager se rattache à l'idéologie du foyer et vise à cultiver la vocation «naturelle» de la femme. Pourtant un grand nombre de jeunes filles issues des classes populaires sont contraintes d'entrer sur le marché du travail afin d'augmenter le modeste revenu familial. Le Canada est d'avis qu'il faudrait songer à ouvrir à leur intention des écoles techniques et des écoles d'arts et métiers, où elles recevraient un apprentissage spécial leur assurant plus tard une occupation décente. Les cours de coupe et de couture organisés dans les couvents lui apparaissent à cet égard comme l'ébauche d'une future école technique «dont le but sera d'outiller d'une façon sérieuse les jeunes filles de condition modeste et qui, au sortir de leurs classes, sont obligées de se chercher un emploi pour soulager le maigre budget de famille». Cette école formerait des «ouvrières d'élite», et «nous ne verrions plus comme aujourd'hui tant de jeunes filles... aller prendre des positions humiliantes et peu rémunératrices, faute d'avoir reçu un enseignement technique» ${ }^{97}$. Afin de faire valoir cette idée, Godfroy Langlois fait paraître dans Le Canada une série d'articles sur le développement de l'enseignement professionnel féminin aux États-Unis 98 .

\footnotetext{
93 «Conseil des Arts et Manufactures», Le Canada, 17 octobre 1903.

94 «Autour de l'école», La Patrie, 20 septembre 1902.

95 «L'enseignement agricole féminin en Belgique», La Patrie, 21 août 1902.

96 «Agriculture-Colonisation: l'agriculture à l'école», Le Canada, 11 avril 1903.

97 «Enseignement plus pratique», ibid., 26 mars 1904.

98 "Quelques écoles de Boston», ibid., 4 avril 1903; «Une école de métiers pour les filles», ibid., 27 novembre 1903.
} 
L'accès des femmes à l'éducation supérieure ne semble guère préoccuper la Ligue de l'Enseignement. Godfroy Langlois aborde indirectement la question dans Le Canada, en se reférant, une fois de plus, à l'exemple des États-Unis. Le journal rapporte l'existence de plusieurs collèges féminins qui dispensent aux jeunes Américaines un enseignement de niveau universitaire conduisant au baccalauréat ès arts. Toutefois, le journal précise bien que ces institutions s'adressent principalement aux filles de la classe bourgeoise. Il faut donc présumer qu'un collège de ce genre établi au Québec accueillerait une clientèle analogue, éventualité que ne conteste pas Le Canada ${ }^{99}$.

En définitive, on se rend compte que relativement à l'éducation des filles, la Ligue de l'Enseignement exprime des convictions qu'on ne peut certes qualifier de progressistes. Ses porte-parole s'abreuvent fondamentalement à l'idéologie conservatrice qui maintient la femme dans le cadre familial. Le type de formation pratique qu'ils proposent est axé sur l'exécution des tâches ménagères nécessaires à la tenue du foyer, et non sur des cours d'apprentissage professionnel. L'idée de préparer les élèves à l'exercice d'un métier n'est envisagée que pour les filles de la classe ouvrière, tandis que l'enseignement supérieur semble réservé aux filles de la bourgeoisie. Bref, la stratification sociale et l'image traditionnelle de la femme se conjuguent lorsqu'il s'agit de déterminer le type d'enseignement souhaité pour les jeunes Québécoises.

\section{$V$ - Réactions du clergé et mise en veilleuse de la Ligue de l'Enseignement}

La présence du groupe radical au sein de la Ligue de l'Enseignement dresse immédiatement le clergé contre cette dernière, même si ses promoteurs affirment avoir invité plusieurs prêtres à joindre les rangs de l'association ${ }^{100}$. Que Godfroy Langlois en soit un des principaux animateurs met évidemment en doute la sincérité de ce geste d'ouverture. D'ailleurs, la Ligue ne tarde pas à obtenir l'appui enthousiaste de journaux «rouges» fort suspects aux yeux du clergé ${ }^{101}$. Le choix du nom donné à la nouvelle association, qui appelle une comparaison immédiate avec son homonyme français, suffit enfin à confirmer les soupçons. D'autant plus que l'Église du Québec a, au même moment, les yeux rivés sur la

\footnotetext{
99 «L'éducation des femmes», ibid., 16 juin 1903; «Éducation de la femme aux États-Unis», ibid., 5 septembre 1903.

100 «Autour de l'école», La Patrie, 10 janvier 1903.

101 Mentionnons L'Union de Saint-Hyacinthe, L'Avenir du Nord de Saint-Jérôme, Le Progrès de Valleyfield, et Les Débats de Montréal, dont le rédacteur, Édouard Charlier, figure sur la liste des membres de la Ligue de l'Enseignement. Le 29 septembre 1903, Les Débats seront frappés d'interdiction par l'archeveque de Montréal, Mgr Bruchési.
} 
France, où le gouvernement d'Émile Combes vient de déclarer la guerre à l'enseignement congréganiste. Appuyé par la francmaçonnerie, son ministère (1902-1905) représente l'apogée de l'anticléricalisme français ${ }^{102}$. Les luttes politico-religieuses françaises agiront donc comme un prisme à travers lequel seront inévitablement jugés le programme et le discours de la Ligue.

L'association avance certes des propositions qui vont à l'encontre de la doctrine de l'Église en matière d'éducation. Soutenir que l'instruction publique est une charge d'État s'oppose directement à la conception cléricale du pouvoir civil, auquel l'Église n'attribue qu'un rôle supplétif. L'oeuvre éducatrice, de par sa nature, relève de la famille et de l'autorité ecclésiastique. L'État doit, pour sa part, seconder et encourager l'initiative privée en apportant son concours financier et en établissant les rouages administratifs nécessaires au bon fonctionnement des écoles. Mais en aucune façon n'a-t-il le droit de s'ériger comme l'éducateur des enfants ${ }^{103}$.

L'avènement de l'État enseignant, tel qu'il s'est institué en France sous Jules Ferry et les Républicains, constitue la principale hantise du clergé québécois. Cette attitude explique pourquoi il condamne vigoureusement toute mesure susceptible, selon lui, de transformer l'enseignement primaire en un service contrôlé entièrement par l'État. C'est ainsi que la gratuité et l'uniformité des livres, réclamées par la Ligue, sont perçues comme le prélude d'un monopole d'État sur les manuels scolaires et, pis encore, de la gratuité générale ${ }^{104}$. L'Église condamne aussi énergiquement l'instruction obligatoire, son argument fondamental étant qu'une telle mesure représente une violation du droit du père à contrôler l'éducation de ses enfants. Et surtout, le clergé québécois voit s'élever derrière la gratuité et l'obligation le spectre de l'école neutre et laïque. Ces trois éléments forment à ses yeux une trilogie indissociable qui, il n'a garde de l'oublier, constitue le couronnement de l'oeuvre anticléricale française. Cet anticléricalisme, il croit le déceler dans le programme de la Ligue, même si ses membres ne s'attaquent jamais ouvertement au caractère confessionnel de l'enseignement au Québec ${ }^{105}$. Enfin, l'Église se méfie en principe de la

${ }_{102}$ Sur cette question, voir Alec Mellor, Histoire de l'anticléricalisme français (Paris, 1966), 381-407.

103 La doctrine officielle de l'Église en matière d'éducation, telle que défendue par le clergé québécois au cours de la période qui nous intéresse, est exposée de façon précise par Mgr Louis-Adolphe Pâquet, dans son ouvrage imposant Droit public de l'Église. L'Église et l'éducation à la lumière de l'histoire et des principes chrétiens (Québec, 1909). Voir en particulier les chapitres 3 et 4: 181-215.

104 Voir, sur cette question, la brochure de l'abbé F.-A. Baillargé, La gratuité des livres (Montréal, 1901).

105 Dans une conférence prononcée au Club Letellier de Montréal, P.-G. Martineau affirmera au contraire que le système éducatif québécois doit conserver sa base religieuse et confessionnelle. Le Canada, 12 mars 1904: Article: «L'instruction publique». 
centralisation scolaire, car elle risque de réduire l'influence des curés dont le pouvoir s'appuie sur la paroisse. Les mesures centralisatrices favorisées par les animateurs de la Ligue au niveau de l'administration scolaire et de la concentration des écoles rurales, ne peuvent qu'accroître la méfiance des autorités religieuses à son endroit ${ }^{106}$.

Afin de soumettre la Ligue au jugement de l'opinion publique, le clergé et la presse catholique ${ }^{107}$ utiliseront la tactique de l'épouvantail en démasquant cette dernière comme une filiale de la Ligue maçonnique française. L'idée d'un complot maçonnique, ourdi par les deux associations contre le système scolaire catholique du Québec, est alors exploitée rondement dans des articles et des brochures, dont certaines connaîtront un succès impressionnant ${ }^{108}$.

L'archevêque de Montréal, Mgr Bruchési, intervient luimême personnellement contre la Ligue. Au début de 1904, il met Napoléon Brisebois en demeure de choisir entre le secrétariat de la Ligue et son poste de professeur à l'École normale JacquesCartier ${ }^{109}$. Mgr Bruchési nourrit d'ailleurs un grief particulier contre Godfroy Langlois, Paul-G. Martineau et d'autres libéraux radicaux de Montréal. Ces derniers mènent à l'époque une campagne soutenue en faveur de la création d'une bibliothèque publique -

${ }^{106}$ Pour un aperçu de l'argumentation tenue par le clergé québécois contre la centralisation scolaire, voir l'article du Père Dominique Gonthier (pseudonyme: Raphaël Gervais), "Erreurs et préjugés», La Nouvelle-France, 4 (1905): 430-438.

107 En particulier La Croix de Montréal et La Vérité de Québec.

108 Tel est le cas de l'ouvrage publié en 1903 par Henri Bernard, un journaliste catholique de naissance française. Portant un titre fort révélateur, La Ligue de l'Enseignement. Histoire d'une conspiration maçonnique à Montréal (Montréal, 1903), il reçoit la haute approbation du Cardinal Merry Del Val, secrétaire d'Etat au Vatican. Épuisé quelques mois à peine après sa publication, l'ouvrage réapparaît en 1904 dans une seconde édition, revue et augmentée. Celle-ci contient une préface rédigée par le comte Albert de Mun, orateur catholique et homme politique français. Le comte félicite Bernard pour son travail contre la Ligue de l'Enseignement. En 1906, un prêtre du diocèse de Québec, l'abbé J.-A. Huot, continue d'alimenter les craintes des fidèles en publiant un ouvrage sur Le fléau maçonnique (Québec, 1906). Le travail est fortement recommandé par l'archevêque de Québec, Mgr Bégin. Voir les Mandements des évêques de Québec, vol. 10, "circulaire au clergé», 15 mai 1906. Les articles parus dans La Semaine religieuse de Montréal et dans La Semaine religieuse de Québec, organes respectifs des archevêchés de Montréal et de Québec, illustrent très bien le type de littérature auquel le clergé a recours pour accréditer la thèse d'un péril maçonnique dans la province de Québec. Voir par exemple, "La francmaçonnerie et les Canadiens français», La Semaine religieuse de Montréal (26 décembre 1904): 425-428; "La franc-maçonnerie chez les Canadiens français», La Semaine religieuse de Québec (17 décembre 1904): 282-284; "Le travail préparatoire de la franc-maçonnerie dans la province de Québec», ibid. (21 janvier 1905): 357-362; "L'influence maçonnique dans la province de Québec», ibid. (1 février 1905): 402.

109 Robert Rumilly, Histoire de la province de Québec, op. cit., 11: 101. Napoléon Brisebois se soumettra aux volontés de Mgr Bruchési et quittera la Ligue de l'Enseignement. 
et neutre - dans la métropole ${ }^{110}$. Or l'archevêque est prêt à remuer ciel et terre pour empêcher l'établissement d'une institution laïque soustraite à la supervision morale de l'Église. Le prélat a donc intérêt à endiguer le mouvement d'opinion qui alimente un tel projet ${ }^{111}$.

Les dénonciations en règle produites contre la Ligue réussissent à semer la confusion au sein de l'association. Celle-ci n'intervient que sporadiquement dans Le Canada pour repousser les allégations concernant son affiliation à la Ligue française de l'Enseignement ${ }^{112}$. À part ces réfutations, elle se réfugie dans un profond mutisme, ce qui n'est pas sans aviver les soupçons du clergé ${ }^{113}$. Au début de 1905, La Semaine religieuse de Québec, organe officieux de l'archevêché, affirme que si la Ligue «n'est pas vraiment morte, [elle] est au moins lourdement endormie» ${ }^{114}$. L'association semble bel et bien avoir été mise en veilleuse; elle ne manifeste dès lors aucun signe d'activité, si bien que des membres du clergé n'hésiteront pas à la considérer comme définitivement enterrée ${ }^{115}$.

Très actifs au sein de la Ligue, les libéraux radicaux constituent donc, paradoxalement, le principal facteur de désintégration de l'association. Les membres modérés ont tôt fait de battre en retraite devant la campagne de censure déclenchée contre l'association. Leur défection, ainsi que les réactions hostiles à la Ligue, démontrent en fait clairement que les réformes scolaires favorisées par les radicaux - ministère de l'Instruction publique, instruction gratuite et obligatoire - ne pouvaient être mises à exécution dans le contexte politico-religieux de l'époque.

Leur programme ne trouve aucune résonance dans les milieux éducatifs catholiques, qui sont bien encadrés par le clergé. Le surintendant de l'Instruction publique, Boucher de la Bruère, est un catholique dévoué aux intérêts de l'Église. En 1902, dans un discours prononcé à Paris lors de la Convention des institutrices, il n'a pas hésité à réprouver la politique de laïcisation poursuivie par le gouvernement français ${ }^{116}$. D'autre part, la doctrine éducative de l'Eglise est prodiguée au corps enseignant par la revue pédagogi-

110 Il est intéressant de noter à ce sujet que la création de bibliothèques populaires constitue l'une des réalisations majeures de la Ligue française de l'Enseignement. Voir Pierre Chevallier, Histoire de la franc-maçonnerie française, op. cit., 3: 34-35.

111 Voir à ce sujet, Th. Jolivet, "Å propos des bibliothèques publiques», La Nouvelle-France (décembre 1902): 560-573.

112 Voir Le Canada, 21 juillet 1903, 4 février 1904, 13 octobre 1904.

113 Voir La Semaine religieuse de Montréal (22 février 1904): 118; La Semaine religieuse de Québec (2 janvier 1904): 320.

114 «Le travail préparatoire de la franc-maçonnerie dans la province de Québec», La Semaine religieuse de Québec (21 janvier 1905): 357.

115 Père Dominique Gonthier, «Erreurs et préjugés», op. cit., 430-431.

116 L'Enseignement primaire (novembre 1902): 153. 
que L'Enseignement primaire, que le gouvernement québécois distribue gratuitement dans toutes les écoles publiques de la province. La revue condamne énergiquement la Ligue et elle met les enseignants en garde contre l'influence pernicieuse des loges maçonniques ${ }^{117}$. Les instituteurs prennent d'ailleurs soin de défendre publiquement le système scolaire québécois contre les critiques que lui a adressées la Ligue ${ }^{118}$. La crainte de se voir soupçonnés de francmaçonnerie prévient malheureusement les éducateurs catholiques d'apprécier à leur juste valeur les éléments constructifs du programme de la Ligue de l'Enseignement.

En réponse aux «détracteurs» du système éducatif catholique, le directeur de L'Enseignement primaire, C.-J. Magnan, publie une brochure imposante dans laquelle il procède à une véritable apologie de l'organisation scolaire au Québec ${ }^{119}$. Magnan dédie son ouvrage à Amédée Robitaille, secrétaire provincial dans le cabinet Parent. Le Soleil, organe des libéraux à Québec, lui réserve un accueil élogieux, aux côtés de l'épiscopat, de la presse catholique et des journaux conservateurs ${ }^{120}$. Un tel alignement révèle que le parti libéral peut difficilement servir de moteur efficace au mouvement radical, qui s'y réfugie pourtant depuis le milieu du XIXe siècle. Déjà, la reculade du gouvernement Marchand sur la question du ministère de l'Instruction publique avait indiqué un recul de la pensée libérale au profit d'une politique de compromis avec le pouvoir ecclésiastique. Les successeurs de Marchand, S.-N. Parent et Lomer Gouin, poursuivront cette politique. Certes, l'arrivée de Gouin au pouvoir en 1905 redonne brièvement espoir aux Rouges. Le nouveau premier ministre est un ami personnel de Godfroy Langlois, dont il a suscité, en 1904, la candidature comme député provincial du comté Saint-Louis à Montréal. On le soupçonne en fait de partager les tendances anticléricales de Langlois et de méditer la laïcisation graduelle de l'enseignement. Mais Gouin, qui tient à maintenir la paix religieuse au Québec, va promettre directement à Mgr Bruchési qu'il s'opposera à tout mouvement de laïcisation ${ }^{121}$. «Nos lois scolaires, déclarera-t-il plus tard, sont aussi bonnes que nous puissions le désirer, et le gouvernement n'a pas l'intention d'en changer les principes.»' 122

117 Ibid. (janvier 1904): 261; (novembre 1904): 171-172; (mars 1905): 435.

118 Voir le compte rendu de la 117 e Conférence de l'Association des instituteurs de l'École normale Jacques-Cartier, 30 mai 1903, dans l'Enseignement primaire (septembre 1903): 14

119 C.-J. Magnan, Honneur à la province de Québec. Mémorial sur l'éducation au Canada (Québec, 1903).

120 L'Enseignement primaire (mai 1903): 515.

121 Robert Rumilly, Histoire de la province de Québec, op. cit., 11: 219-220.

122 Ibid., 12: 95. 
La nécessité de composer avec le clergé amène donc Gouin à renier le programme éducatif des libéraux avancés. Élu député en 1904, Godfroy Langlois fera cavalier seul à chaque fois qu'il réclamera à l'Assemblée législative les réformes prônées jadis sous une forme détournée par la Ligue de l'Enseignement: contrôle de l'État sur l'éducation par l'entremise d'un ministère de l'Instruction publique, instruction gratuite et obligatoire. Marginalisé au sein du gouvernement et du parti libéral, le groupe radical effectuera un rapprochement avec les syndicats internationaux qui revendiquent à l'époque les mêmes mesures. Ces organisations ouvrières comptent parmi leurs leaders des éléments taxés d'anticléricalisme par le clergé, tel le socialiste Gustave Francq, un partisan notoire de l'instruction gratuite et obligatoire ${ }^{123}$. Cette collusion des principes «rouges» avec les réclamations du syndicalisme international neutre ne pouvait qu'attiser la méfiance de l'Église et écarter davantage les radicaux du pouvoir ${ }^{124}$.

\section{$V I$ - Bilan de l'évolution scolaire au Québec au début du XXe siècle}

La mise en veilleuse de la Ligue de l'Enseignement après une existence plutôt brève ne doit pas minimiser l'importance du courant d'opinion qui lui a donné naissance. En peu de temps, la Ligue a obtenu l'adhésion de membres influents de la bourgeoisie libérale, détenant, nous l'avons vu, des postes d'autorité dans la presse à grand tirage, dans la politique et dans les affaires. Au début du XXe siècle cette élite bourgeoise est consciente que le système scolaire souffre de carences importantes et qu'il est nécessaire de l'adapter aux besoins nouveaux de l'heure. L'amélioration de l'enseignement primaire s'impose avant tout, car c'est à l'école publique que la majorité des enfants auront l'unique opportunité d'acquérir l'instruction de base nécessaire, d'une part, au perfectionnement des pratiques agricoles, et, d'autre part, à la formation subséquente d'une main-d'oeuvre spécialisée dans les écoles techniques. La campagne intensive que mènent les animateurs de la Ligue dans La Patrie et dans Le Canada fait écho à cette prise de conscience. Les idées véhiculées dans les colonnes de la presse libé-

123 Sur les revendications ouvrières en matière d'éducation, voir Jacques Rouillard, "L'action politique ouvrière au début du 20e siècle», dans Fernand Harvey, éd., Le mouvement ouvrier au Québec (Montréal, 1980), 185-213. Voir aussi Arthur Saint-Pierre, L'organisation ouvrière dans la province de Québec (Montréal, École sociale populaire, 1913).

124 Le sort réservé à Godfroy Langlois met en évidence la marginalisation de l'aile gauche libérale au sein du gouvernement Gouin. En 1910, suite à une intervention de Mgr Bruchési, le premier ministre invite Langlois à quitter son poste de rédacteur du Canada. Puis, en 1914, Langlois est définitivement écarté lorsque Gouin le nomme agent de la province de Québec à Bruxelles. Le groupe radical venait ainsi de perdre sa principale tête d'affiche. 
rale sont appelées à se répandre, si ce n'est à peser au sein des centres de décision responsables de la formulation des politiques éducatives. En fait, un bref aperçu de l'évolution scolaire au Québec au début du XXe siècle démontre que, hormis les réformes controversées privilégiées par les radicaux, plusieurs éléments du programme proposé par la Ligue et ses porte-parole furent réalisés au cours de cette période, et plus précisément sous le gouvernement Gouin (1905-1920).

Même s'il repousse les demandes des Rouges, Lomer Gouin donne, en effet, une impulsion nouvelle à la cause de l'éducation. Le premier ministre soutient en particulier que «le premier et le meilleur emploi» que l'État puisse faire de ses recettes, «c'est de subventionner largement les écoles» ${ }^{125}$. C'est justement en pratiquant une politique financière active que l'administration Gouin exercera principalement son action dans le domaine éducatif. Ainsi, les sommes affectées au soutien de l'éducation triplent entre 1906 et $1916^{126}$. Une part importante des octrois est consacrée à l'enseignement primaire, auquel le gouvernement accorde une attention spéciale. Les subventions se répartissent de multiples façons: hausse du fonds annuel voté pour les écoles primaires, primes aux municipalités scolaires qui paient convenablement le personnel enseignant, primes aux institutrices et aux instituteurs jugés les plus méritants, augmentation du traitement des inspecteurs, etc. Enfin, le gouvernement Gouin subventionne la fondation de neuf Écoles normales de filles, dont il confie la direction à des communautés religieuses.

Le problème des écoles rurales retient aussi l'attention du gouvernement Gouin. Afin de pourvoir les campagnes d'enseignants qualifiés, un crédit spécial est réservé aux municipalités rurales qui accordent aux instituteurs et aux institutrices un traitement minimum fixé par le gouvernement. De plus, le ministère québécois de l'Agriculture encourage le développement de l'enseignement agricole dans les écoles rurales en subventionnant et en organisant l'oeuvre des jardins scolaires. C'est surtout grâce aux efforts d'un ancien membre de la Ligue de l'Enseignement, O.-E. Dalaire, que ce mouvement prend son élan à partir de 1905. Alors que onze écoles seulement tentent l'expérience des jardins scolaires

125 Discours prononcé par Lomer Gouin à l'École Montcalm de Montréal le 5 avril 1905, dans Jean-Louis Roy, Les programmes électoraux du Québec (Montréal, 1970), 1: 128-129.

126 Totalisant 536100 \& en 1906, la somme globale des octrois gouvernementaux affectés à l'éducation atteint $1782417 \$$ en 1915. En 1920, ces octrois s'élèveront à 2334108 \$. Québec, Département de l'Instruction publique, Statistiques de l'enseignement, 1920-1921: 222, tableau XVII. 
en 1905 , on en compte 188 en 1910 et plus de 1468 en $1920^{127}$. D'autres initiatives viendront éventuellement s'ajouter à cette entreprise: cours agricoles d'été pour les enseignants et les inspecteurs d'écoles, cours régionaux d'agriculture dispensés aux institutrices rurales, expositions scolaires ${ }^{128}$.

C'est toutefois la création d'un réseau d'écoles techniques et professionnelles qui constitue la réalisation la plus imposante de l'administration Gouin. Avant la mise sur pied en 1910 d'une Commission royale d'enquête sur l'enseignement industriel et technique, Lomer Gouin perçoit déjà la nécessité d'adapter le système scolaire aux exigences nouvelles de l'économie. Le Premier ministre tient à cet égard un discours similaire à celui véhiculé par les membres de la Ligue de l'Enseignement. Permettre aux Canadiens français d'accéder aux carrières industrielles et commerciales, et procurer en même temps aux manufacturiers des «chefs d'atelier émérites, des contremaîtres expérimentés et des ouvriers d'élite», tels sont les objectifs que veut atteindre le gouvernement libéral en organisant l'enseignement professionnel au Québec ${ }^{129}$. Cette prise de position donne lieu à plusieurs mesures importantes: assistance accrue à l'École polytechnique de Montréal (fondée en 1873), création d'une École d'arpentage (1907) et d'une École forestière (1910), rattachées toutes deux à l'Université Laval, et surtout, fondation en 1907 d'une École des Hautes Études commerciales et.des Écoles techniques de Montréal et de Québec ${ }^{130}$. Conformément au désir exprimé par les membres de la Ligue, l'État québécois prend donc les devants et oriente le système éducatif hors des sentiers battus.

Au cours de la même période, le Comité catholique du Conseil de l'Instruction publique tente d'apporter des correctifs à quelques-uns des principaux problèmes relevés par la Ligue de l'Enseignement dans le domaine de l'instruction primaire. Afin de pallier la faible préparation des institutrices, le Comité crée à leur intention des congrès pédagogiques qui se tiennent annuellement dans les différents diocèses de la province. Il demande aussi (sans succès) au gouvernement de fixer un salaire minimum provincial

${ }^{127}$ Rapport du surintendant de !'Instruction publique, 1904-1905: ix. Québec, Département de l'Instruction publique, Statistiques de l'enseignement, 1920-1921: 219, tableau XIII.

128 Jean-Charles Magnan, Souvenirs. Fleurs et chardons (Saint-Romuald, Québec, Éditions Etchemins, 1976), 29-30.

${ }_{129}$ Voir, à ce sujet, le discours prononcé par Lomer Gouin à l'Assemblée législative le 8 mars 1907 et reproduit dans Le Soleil du 9 mars 1907.

130 C'est un membre influent de la Ligue de l'Enseignement, le capitaliste Gaspard de Serres, qui deviendra le premier président de l'École technique de Montréal. En 1910, il sera nommé membre de la Commission royale d'enquête sur l'enseignement industriel et technique, présidée par J. W. Robertson, avocat et principal du MacDonald College. Voir J.-A. Buteau, Notre enseignement technique industriel (Québec, 1919), 24-25. 
pour l'ensemble du corps enseignant. Il obtient toutefois de l'administration Gouin l'augmentation du nombre des inspecteurs, de même que la nomination d'un inspecteur général. D'autre part, le Comité catholique se préoccupe d'améliorer l'enseignement de l'hygiène dans les écoles et cherche aussi à répandre l'éducation physique en participant à la distribution du fonds créé à cet effet par lord Stratchona. Au niveau de la classification des écoles, le Comité intègre en 1911 la catégorie des écoles maternelles aux structures scolaires existantes.

Responsable de l'élaboration des programmes d'études, le Comité catholique manifeste enfin le désir d'adapter l'enseignement primaire aux besoins du jour en lui imprimant un caractère pratique et utilitaire. En 1905, le Comité inscrit pour la première fois au programme des cours de sciences usuelles basés sur des leçons de choses, ainsi que des cours d'économie domestique pour les filles ${ }^{131}$. En 1912, il adopte un nouveau programme de l'enseignement du dessin ayant pour fin «de faire acquérir des connaissances et des pratiques techniques utiles à tous et indispensables aux carrières industrielles, aux métiers et aux travaux manuels» ${ }^{132}$. Cette orientation pratique conférée au programme d'études se reflète dans les directives pédagogiques qui s'y rattachent. Celles-ci condamnent en effet la mémorisation excessive et l'utilisation exclusive du manuel; elles recommandent plutôt le procédé intuitif dont «l'emploi est indispensable aux enfants, qui ont pris, jusquelà, toutes leurs connaissances dans le monde concret de leur entourage» ${ }^{133}$. Le programme d'études qu'adopte le Comité en 1921 (il ne sera mis en vigueur qu'en 1923) illustre encore plus clairement la direction qu'on veut donner à la formation des élèves. Il introduit, au niveau de la $7 \mathrm{e}$ et de la $8 \mathrm{e}$ année, un cours complémentaire divisé en quatre sections - agricole, commerciale, industrielle, ménagère - qui ajoutent à l'instruction générale «quelques essais de préapprentissage destinés à orienter les élèves vers les métiers ou professions manuelles» ${ }^{134}$. Il est spécifié aussi que le travail manuel doit prévaloir au niveau des méthodes et que les maîtres «feront toujours passer la pratique avant la théorie» "135. Enfin, il est prévu que l'orientation des élèves se conformera à leur milieu social. Les sections industrielle et commerciale sont ainsi destinées aux écoles urbaines de garçons, et la section agricole, aux écoles

\footnotetext{
${ }^{131}$ Rapport du surintendant de l'Instruction publique, 1904-1905: viii.

132 Ibid., 1912-1913: xi.

133 Programme d'études de l'école primaire élémentaire (Québec, 1920), 12.

134 Rapport présenté au Comité catholique en mai 1921 par l'abbé Adélard Desrosiers, rapporteur du sous-comité du programme d'études, dans L'Enseignement primaire (avril 1922): 472.

135 Ibid.: 135.
} 
rurales de garçons. Les matières comprises dans la section ménagère varieront aussi suivant le milieu urbain ou rural.

Les programmes d'étudés insistent particulièrement sur l'importance de l'enseignement agricole dans les milieux ruraux. Le programme de 1923 l'impose comme «une nécessité sociale» dont le but premier est d'inculquer aux élèves «des convictions solides sur l'importance et la noblesse de l'agriculture» ${ }^{136}$. Il va sans dire que le Comité catholique, le surintendant de l'Instruction publique et le Département de l'Instruction publique appuient inconditionnellement les démarches effectuées à l'époque par le ministère de l'Agriculture dans le but de «ruraliser» l'enseignement primaire ${ }^{137}$.

Enfin, au cours de la période qui nous intéresse (1905-1920), l'éducation des filles évolue largement dans la direction souhaitée par la Ligue de l'Enseignement. L'enseignement ménager connaît en effet un développement rapide et devient le type de formation féminine le plus populaire de l'époque. Comme nous l'avons mentionné, des cours d'économie domestique sont introduits en 1905 dans le programme d'études primaires, et en 1923, une section ménagère obligatoire est incluse dans le programme du nouveau cours complémentaire. Par ailleurs, les écoles ménagères se multiplient à partir de 1905 sous l'égide du ministère québécois de l'Agriculture. Alors qu'il n'en existe que deux en 1905, on en compte 59 en $1920^{138}$. Subventionnées par le gouvernement, elles sont dirigées toutefois par des communautés religieuses qui les établissent dans leurs couvents. Ces institutions sont vouées pour la plupart à l'enseignement ménager agricole. Par ailleurs, des laïques appartenant à la section féminine de la Société Saint-JeanBaptiste de Montréal ouvrent en 1906 la première école ménagère de la métropole. L'idée d'une telle fondation a été conçue en 1904 par un groupe de bourgeoises comprenant, entre autres, Mesdames F.-L. Béique, Raoul Dandurand et Damien Rolland, dont les époux ont adhéré à la Ligue de l'Enseignement. Joseph-Israël Tarte, Gaspard de Serres et d'autres membres de la Ligue ont également endossé le projet à l'époque ${ }^{139}$. À l'instar des porte-parole masculins de la Ligue, ces femmes assignent à l'enseignement ménager des visées traditionnelles: former des épouses et des femmes d'intérieur à la hauteur de leur tâche, sans empiéter sur les

\footnotetext{
${ }_{136}$ Règlements du Comité catholique du Conseil de l'Instruction publique de la province de Québec (Québec, 1924), 148.

137 Voir par exemple, Rapport du Surintendant de l'instruction publique, 19011902: xvii-xx; 1912-1913: xiii-xiv; 1917-1918, «Circulaires»: 431-432.

${ }_{138}$ Le Gouvernement Gouin et son oeuvre (Québec, 1916), 109; Québec, Département de l'Instruction publique, Statistiques de l'enseignement, 1920-1921: 219, tableau XIII.

${ }_{139}$ Mme F.-L. Béïque, Quatre-vingts ans de souvenirs (Montréal, 1939), 244-245.
} 
droits de l'homme ${ }^{140}$. Sur la question de l'enseignement ménager, la bourgeoisie canadienne-française se rallie donc à l'idéologie conservatrice véhiculée par le clergé et les milieux éducatifs catholiques $^{141}$.

Les deux autres avenues éducatives envisagées par Godfroy Langlois pour les jeunes Québécoises, soit l'enseignement professionnel et l'enseignement supérieur, se développent par contre timidement au début du siècle. Le gouvernement Gouin subventionne des cours de coupe et de couture qui se donnent gratuitement le soir dans les principaux centres du Québec. L'augmentation croissante du nombre des classes et des inscriptions semble indiquer que ce type d'enseignement répond à un besoin réel ${ }^{142}$. D'autre part, l'enseignement supérieur féminin s'organise sous la supervision étroite de l'Église. En 1908, la Congrégation NotreDame de Montréal ouvre le premier collège classique féminin, dont le cours permet l'accès aux études universitaires. Cette institution s'adresse manifestement aux filles de la bourgeoisie et elle n'attire à ses débuts qu'un nombre infime d'élèves ${ }^{143}$. Selon l'archevêque de Montréal, Mgr Bruchési, l'enseignement supérieur féminin est d'ailleurs réservé à une «élite» qui, après avoir acquis la «science chrétienne», sera en mesure d'exercer «une forte et salutaire influence» dans le milieu où elle sera appelée à vivre. Ainsi, il n'est toujours pas question pour la femme de sortir de son élément «naturel» ${ }^{144}$.

\section{Conclusion}

Le bilan qui précède démontre que le gouvernement libéral de Lomer Gouin et les milieux éducatifs catholiques sont conscients, au début du XXe siècle, que l'industrialisation et l'urbanisation ont créé des conditions nouvelles qui imposent des changements au système éducatif. Leur action se traduit par une série de mesures qui marquent largement l'évolution de l'enseignement au Québec au cours de cette période. Ces mesures comprennent, entre autres,

140 Voir par exemple la «Conférence sur l'enseignement ménager, par Mademoiselle Gérin-Lajoie, directrice de l'École ménagère provinciale», dans Premier Congrès de la Fédération nationale Saint-Jean-Baptiste, tenu les 26, 27, 28, 29 mai à Montréal, (Montréal, 1907), 126.

${ }_{141}$ Voir par exemple le discours prononcé par l'abbé Perrier, dans ibid., 74-77. Voir aussi les propos du Surintendant de l'instruction publique, Pierre Boucher de La Bruère, dans le Rapport du Surintendant de l'instruction publique, 1908-1909: xx-xxvii.

142 Pour l'année 1902-1921, les statistiques rapportent 2347 élèves inscrites aux 58 cours de coupe et de couture organisés à travers la province. Québec, Département de l'Instruction publique, Statistiques de l'enseignement, 1920-1921: 220, tableau XV.

${ }_{143}$ Robert Rumilly, Histoire de la province de Québec, op. cit., 16: 153.

144 Lettre de Mgr Bruchési à la révérende Mère Sainte-Euphrosyne, maitresse générale des études, Congrégation Notre-Dame, 16 juin 1908, dans La Semaine Religieuse de Montréal (20 juin 1908): 391. 
la révision du programme d'études primaires et son orientation vers un enseignement plus pratique, la création d'écoles techniques et professionnelles, l'organisation de l'enseignement ménager et la «ruralisation» de l'enseignement dans les centres agricoles. À cet égard, le Québec opte pour des politiques éducatives comparables à celles mises en application par la province voisine, l'Ontario, où les promoteurs de l'«éducation nouvelle» sont particulièrement actifs. La similitude des problèmes et des besoins créés par le développement industriel et urbain amène donc l'adoption de solutions analogues, d'où l'importance d'analyser l'expérience éducative québécoise dans une perspective comparative. Une telle approche permet de repérer des modèles communs de pensée et d'action, de mesurer la fréquence et l'étendue des échanges et d'évaluer l'apport des influences extérieures, contribuant ainsi à éclairer davantage la spécificité du cas québécois.

$\mathrm{Au}$ début du XXe siècle, cette spécificité repose principalement sur l'absence d'un système scolaire gratuit, obligatoire et régi par un ministère d'État. De plus, le principe traditionnel de l'autonomie locale continue à prévaloir, posant toujours un obstacle à la centralisation administrative et financière du système éducatif. Enfin, l'importance du rôle joué par l'Église dans le champ scolaire explique la présence au Québec d'un courant radical teinté d'anticléricalisme qui épouse largement les idéaux de la France républicaine. Comme nous l'avons vu, la désagrégation de la Ligue de l'Enseignement indique qu'à peine quelques années après la tentative du gouvernement Marchand pour rétablir le ministère de l'Instruction publique, la conjoncture n'était guère plus favorable à ce courant dirigé par Godfroy Langlois qu'elle ne l'avait été, un demi-siècle plus tôt, au «rougisme» dont il était l'héritier. 